\title{
Basic FBG apodization functions effects on the filtered optical acoustic signal
}

\author{
Mahmoud M. A. Eid ${ }^{1}$, Ahmed Nabih Zaki Rashed ${ }^{2}$ \\ ${ }^{1}$ Department of Electrical Engineering, College of Engineering, Taif University, Kingdom of Saudi Arabia \\ ${ }^{2}$ Department of Electronics and Electrical Communications Engineering, Faculty of Electronic Engineering, \\ Menoufia University, Egypt
}

\begin{tabular}{l} 
Article Info \\
\hline Article history: \\
Received Sep 16, 2020 \\
Revised Dec 8, 2020 \\
Accepted Jan 6, 2021 \\
\hline Keywords: \\
Acoustic signal \\
Gaussian apodization \\
Tanh apodization \\
Uniform apodization
\end{tabular}

\begin{abstract}
This article clarified the basic fiber bragg grating (FBG) apodization functions effects on the filtered optical acoustic signal (AS). Max optical acoustic power variations after acousto optic filter is clarified with the spectral wavelength variations. FBG apodization functions are uniform, Gaussian, and Tanh. We have tested the max optical acoustic power variations after various apodization FBG functions with the spectral wavelength variations. The max AS power amplitude after the electrical combiner is reported based various apodization FBG functions. The max optical AS after FBG is studied with various FBG lengths for various FBG apodization functions at the central wavelength of $1.55 \mu \mathrm{m}$. The max electrical power after power combiner unit is demonstrated with different FBG lengths for various FBG apodization functions at the central frequency of $193.1 \mathrm{THz}$.
\end{abstract}

This is an open access article under the $\underline{C C B Y-S A}$ license.

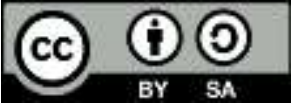

\section{Corresponding Author:}

Ahmed Nabih Zaki Rashed

Faculty of Electronic Engineering

Menoufia University

Egypt

E-mail: ahmed_733@yahoo.com

\section{INTRODUCTION BACKGROUND}

Optical communication system should satisfy the following basic specifications such as determination of transmission type (analog/digital); achieving the system fidelity (higher SNR/lower BER); larger transmission bandwidth (BW); longer repeater spacing (span); less cost of system installation; reliability (error-free transmission); less cost of system maintenance; and less of complexity [1-15]. While selecting a photodetector, the following factors are considered such as minimum optical power that must fall on photodiode to satisfy BER at specified data rate; less complexity of circuit; less cost of design; and less bias requirements [16-35]. Metal oxide technology is used to reduce the rise, fall and delay times. Metal oxide technology can be employed to achieve high speed performance, minimize transistor dimensions, so large transistors can be integrated on achip. The progress of transistors integrated on a single chip versus technology year can be studied in these researches. Bipolar complentary semiconductor transistors technologies are integrated with metal oxide technology to overcome the time delay and consequently to increase the flow rates. Next step in system designing is choosing a proper optical source, the important factors are less signal dispersion; higher data rate; longer transmission distance; less cost; efficient optical power coupling; and less circuit complexity [36-58]. 


\section{MODEL DESCRIPTION}

The setup model is illustrated in Figure 1. WDM transmitter has $100 \mathrm{GHz}$ frequency spacing, power of $0 \mathrm{dBm}$, modulation line coding of non return to zero and flow rate of $40 \mathrm{Gbps}$. WDM transmitter generates the light signal with the previous technical specifications. The light signal can be modulated through acousto optical filter to delete the unwanted noise from the base band. The filtered AS is forward to FGB device to smooth the filtered signal. Fiber bragg grating (FBG) has input frequency of $193.1 \mathrm{THz}$. The Laser measured has $193.1 \mathrm{THz}$ frequency of and input power of $10 \mathrm{dBm}$. SGs generate bits stream and it is digitalized coding through NRZ line coding modulation scheme. The encoded light signal from laser measured is directed to the ideal circulator. Ideal circulator has two inputs, one is the filtered AS through FBG devices and another is the encoded light signal. The output of the ideal circulator is directed to APD photodetectors.

The basic function of APD photodetectors is electrical signal detection. The signal is amplified through transimpedance amplifiers. Transimpedance amplifiers have voltage gain of $600 \mathrm{ohm}$ and noise figure of $6 \mathrm{~dB}$. The two signals with amplification are combined together in order to be measured in the time and spectral domains.

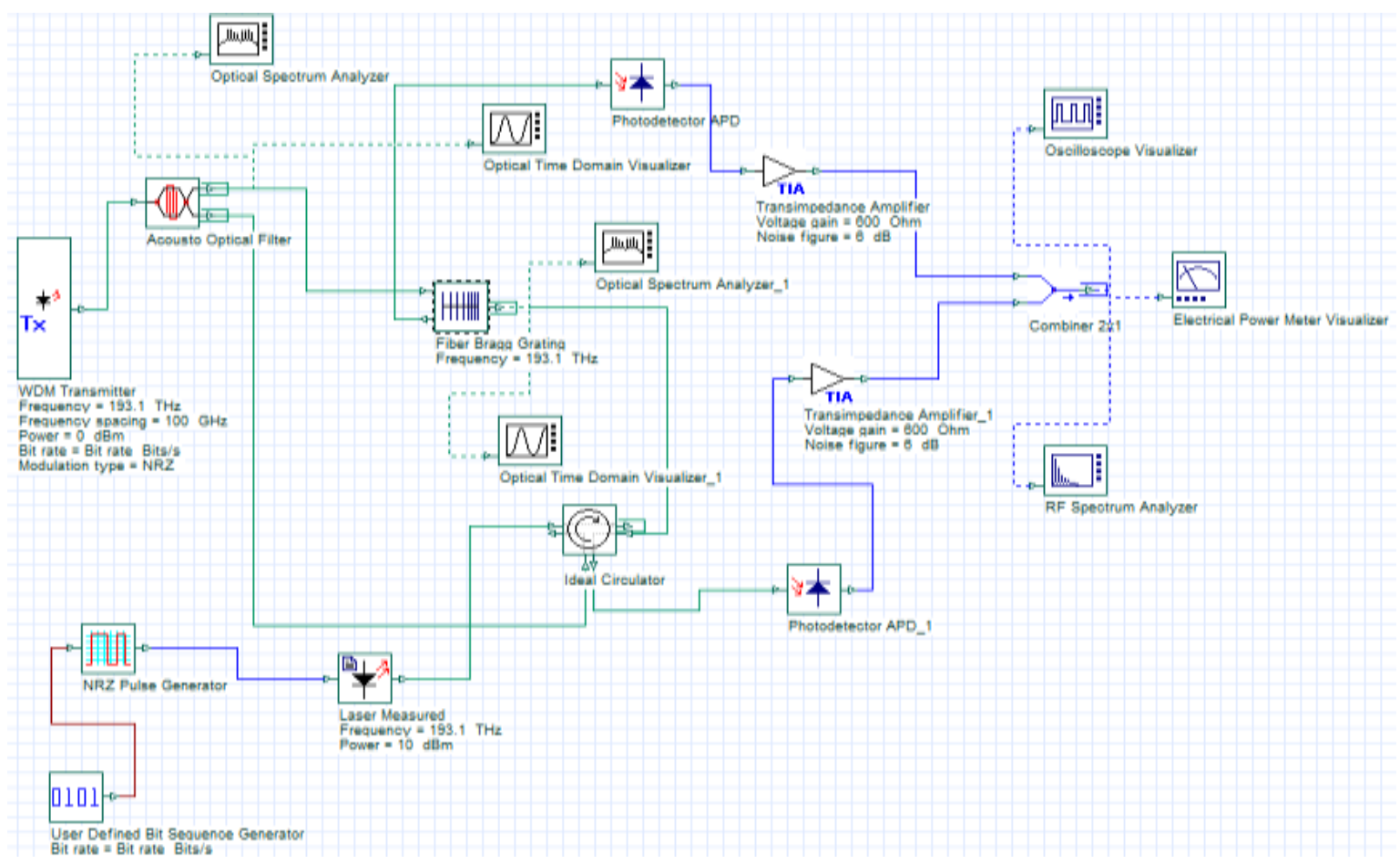

Figure 1. Proposed simulation setup description

\section{PERFORMANCE ANALYSIS WITH DISCUSSIONS}

We have outlined the basic fiber bragg grating (FBG) apodization function (AF) effects on the filtered optical AS. We have also studied the max optical acoustic power variations (PVs) after acousto optic filter with the spectral wavelength variations. Various FBG apodization functions are studied such as the uniform, Gaussian, and Tanh. We have examined the max optical acoustic PVs after various apodization FBG functions with the spectral wavelength variations. We have simulated the max AS power amplitude after the electrical combiner based various apodization FBG functions. The max optical AS after FBG is simulated also with various FBG lengths for various FBG apodization functions at the central wavelength of $1.55 \mu \mathrm{m}$. The max electrical power after power combiner unit is demonstrated with different FBG lengths for various FBG apodization functions at the central frequency of $193.1 \mathrm{THz}$. list of variables in this proposed study as shown in Table 1.

Figure 2 clarifies the max optical acoustic PVs after acousto optic filter with the spectral wavelength variations. Where max AS power is $0.5205 \mathrm{dBm}$ and min noise acoustic power (MNAP) is $-104.787 \mathrm{dBm}$. The max optical acoustic PVs after uniform apodization FBG with the spectral wavelength variations is reported in Figure 3. Where the max AS power is $-0.206788 \mathrm{dBm}$ and MNAP is $-104.752 \mathrm{dBm}$. Figure 4 shows the max optical acoustic PVs after Gaussian apodization FBG with the spectral wavelength variations. 
Where the max AS power is $0.318 \mathrm{dBm}$ and MNAP is $-104.777 \mathrm{dBm}$. The max optical acoustic power variations after Tanh apodization FBG with the spectral wavelength variations is reported in Figure 5. Where the max AS power is $-0.17823 \mathrm{dBm}$ and MNAP is $-104.753 \mathrm{dBm}$.

Table 1. List of variables in this proposed study

\begin{tabular}{cc}
\hline Parameters & Values/Units \\
\hline \multicolumn{2}{c}{ WDM transmitter } \\
Frequency & $193.1 \mathrm{THz}$ \\
Power & $0 \mathrm{dBm}$ \\
Frequency spacing & $100 \mathrm{GHz}$ \\
Extinction ratio & $10 \mathrm{~dB}$ \\
Acousto opticl & filter \\
Bandwidth & $100 \mathrm{GHz}$ \\
Insertion loss & $0 \mathrm{~dB}$ \\
Laser measured \\
Frequency & $193.1 \mathrm{THz}$ \\
Power & $10 \mathrm{dBm}$ \\
\multicolumn{2}{c}{ FGB Device } \\
Frequency & $193.1 \mathrm{THz}$ \\
Length & $2 \mathrm{~mm}$ \\
Effective index & 1.45 \\
Transimpedance & Amplifier \\
Voltage gain & $27.78 \mathrm{~dB}$ \\
Noise figure & $6 \mathrm{~dB}$ \\
\hline \multicolumn{2}{c}{}
\end{tabular}
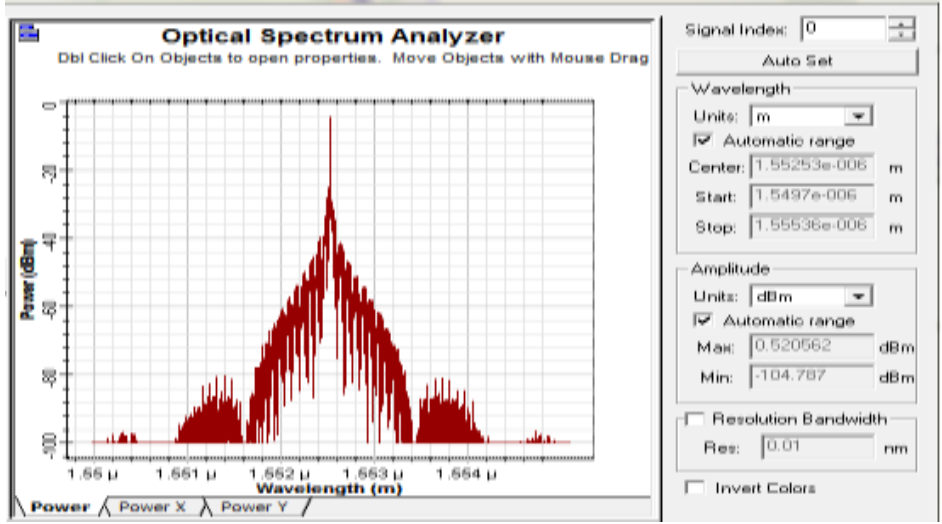

Figure 2. Max optical acoustic power variations after acousto optic filter with the spectral wavelength variations
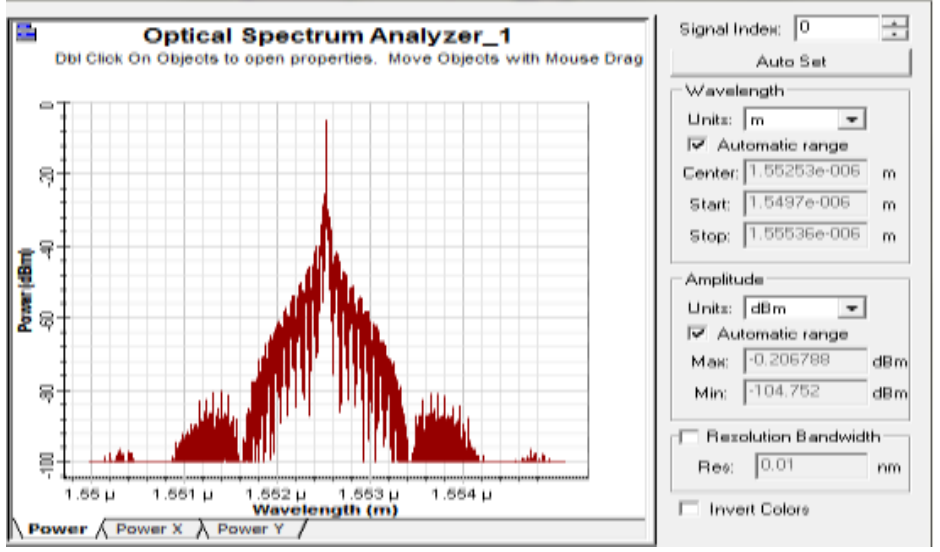

Figure 3. Max optical acoustic power variations after uniform apodization FBG with the spectral wavelength variations 


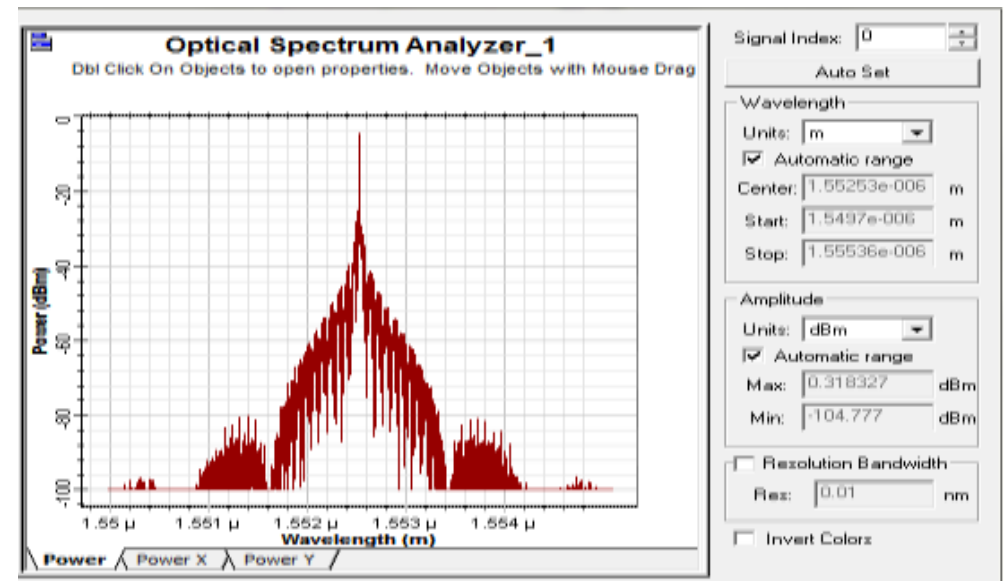

Figure 4. Max optical acoustic power variations after Gaussian apodization FBG with the spectral wavelength variations

Figure 6 demonstrates the max optical acoustic power variations (OAPVs) after Acousto Optic filter with the time variations. Where the max acoustic power is $0.00105 \mathrm{~W}$ and MNAP is $-50 \mu \mathrm{W}$. The max OAPVs after uniform apodization FBG with the time variations is reported in Figure 7. Where the max acoustic power is $0.00092 \mathrm{~W}$ and MNAP is $-43.85 \mu \mathrm{W}$. Besides the max OAPVs after Gaussian apodization FBG with the time variations is clarified in Figure 8. Where the max acoustic power is $0.001 \mathrm{~W}$ and MNAP is $-47.93 \mu \mathrm{W}$. Figure 9 outlines the max OAPVs after Tanh apodization FBG with the time variations. Where the max acoustic power is $0.00095 \mathrm{~W}$ and min acoustic power is $-44.05 \mu \mathrm{W}$. Figure 10 shows the max AS power amplitude after the electrical combiner based Gaussian apodization FBG. The max AS amplitude is 1.54958 a.u. and min AS amplitude is -0.276726 a.u. The total electrical power after the electrical combiner based Gaussian apodization FBG is demonstrated in Figure 11. Where the total AS power is $806.165 \mathrm{~mW}$.

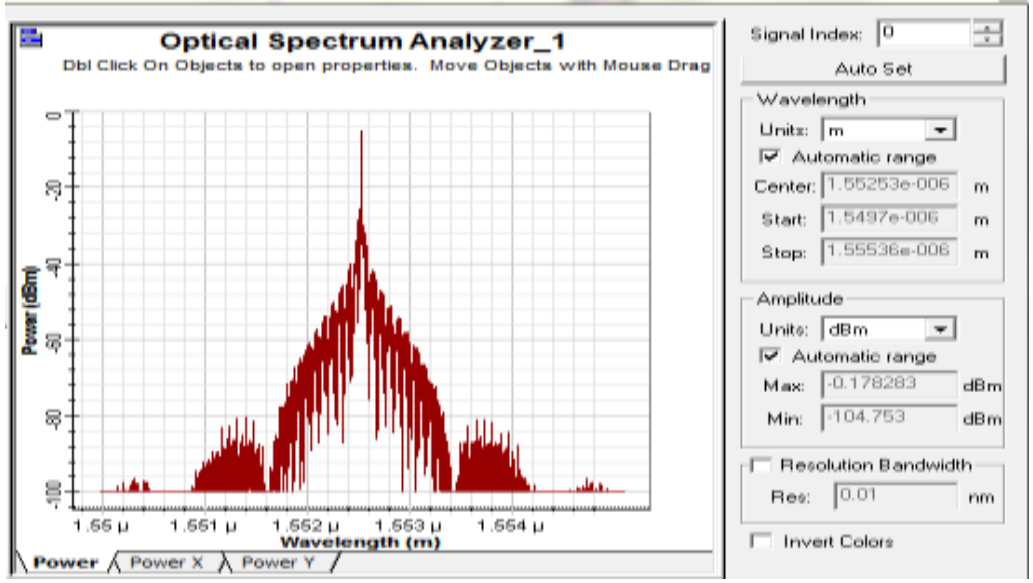

Figure 5. Max optical acoustic power variations after Tanh apodization FBG with the spectral wavelength variations

Figure 12 clarifies the max electrical power variations with the spectral frequency variations after the electrical combiner based Gaussian apodization FBG. Where the max AS power is $33.2231 \mathrm{dBm}$ and min MNAP is $-106.344 \mathrm{dBm}$. Figure 13 clarifies the max optical AS after FBG with FBG length for various FBG $\mathrm{AF}$ at $1.55 \mu \mathrm{m}$ center of wavelength. The max optical AS decreases with the increase of FBG device length. The optimum optical AS is achieved at FBG length of $2 \mathrm{~mm}$ for various FBG AF. Gaussian AF reported better performance than other apodization FBG AF. Figure 14 illustrates the max AS power amplitude after power combiner unit with FBG length for various FBG AF at the central wavelength of $1.55 \mu \mathrm{m}$. The max AS power amplitude can be enhanced with FBG length of $2 \mathrm{~mm}$ for various FBG AF. Gaussian FBG AF has outlined better performance than other FBG AF. 


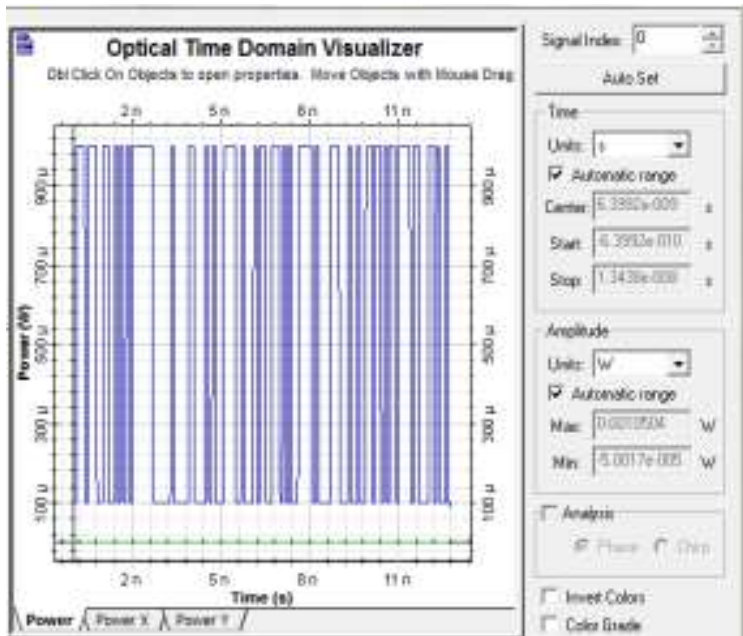

Figure 6. Max optical acoustic power variations after Acousto Optic filter with the time variations

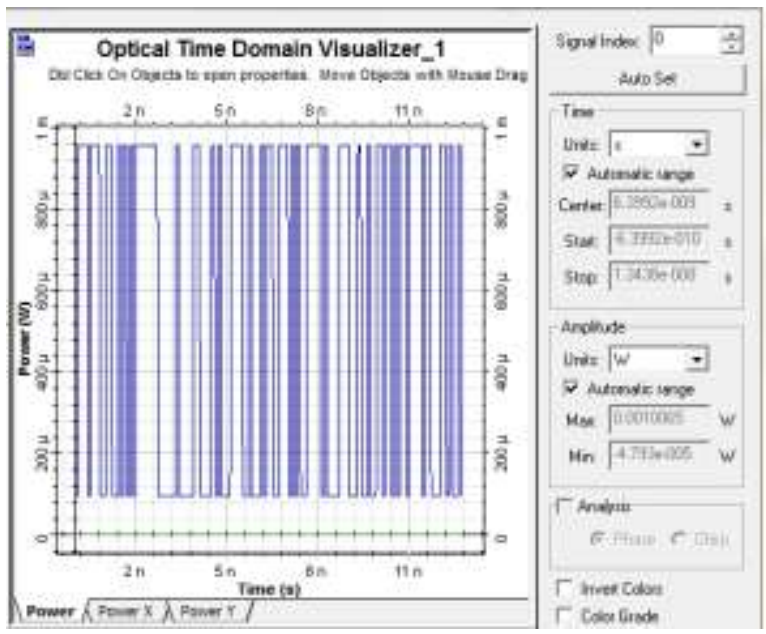

Figure 8. Max optical acoustic power variations after Gaussian apodization FBG with the time variations

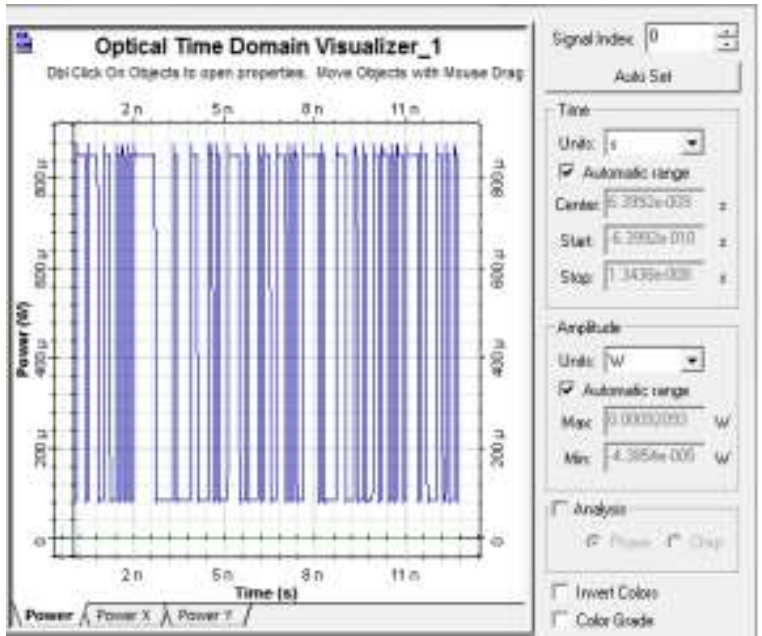

Figure 7. Max optical acoustic power variations after uniform apodization FBG with the time variations

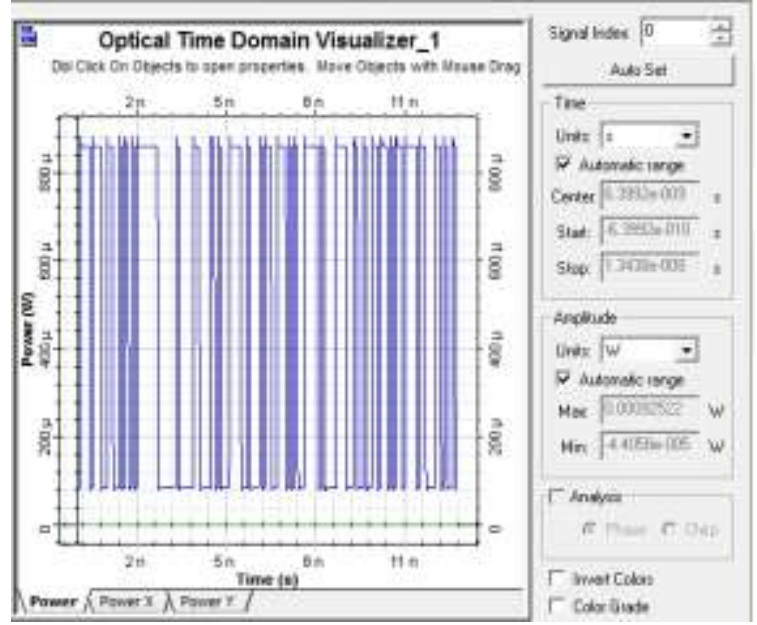

Figure 9. Max optical acoustic power variations after Tanh apodization FBG with the time variations

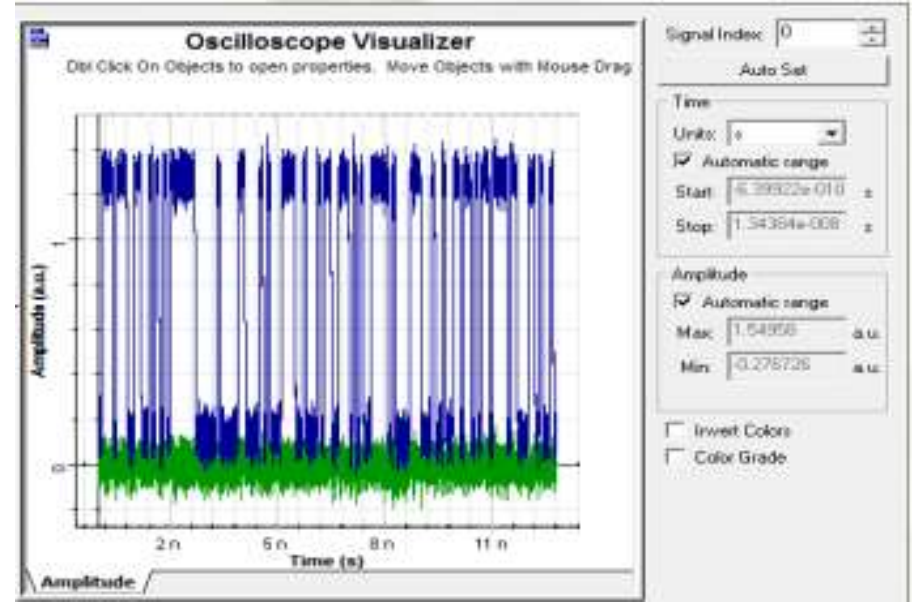

Figure 10. Max AS power amplitude after the electrical combiner based Gaussian apodization FBG 


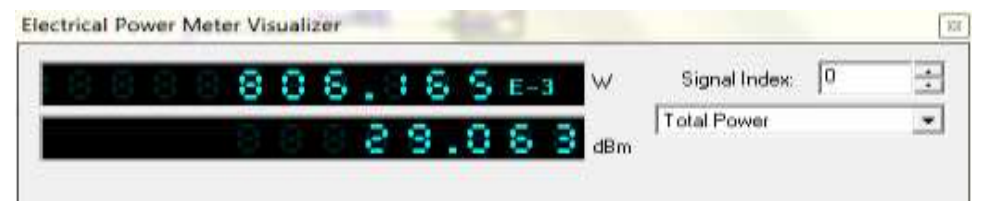

Figure 11. Total electrical power after the electrical combiner based Gaussian apodization FBG

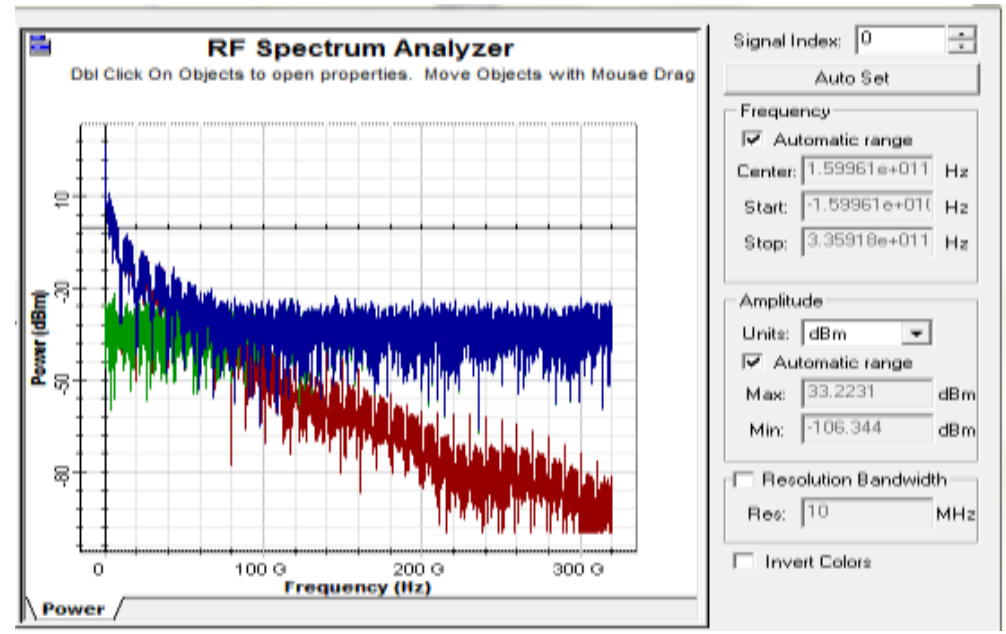

Figure 12. Max electrical power variations with the spectral frequency variations after the electrical combiner based Gaussian apodization FBG

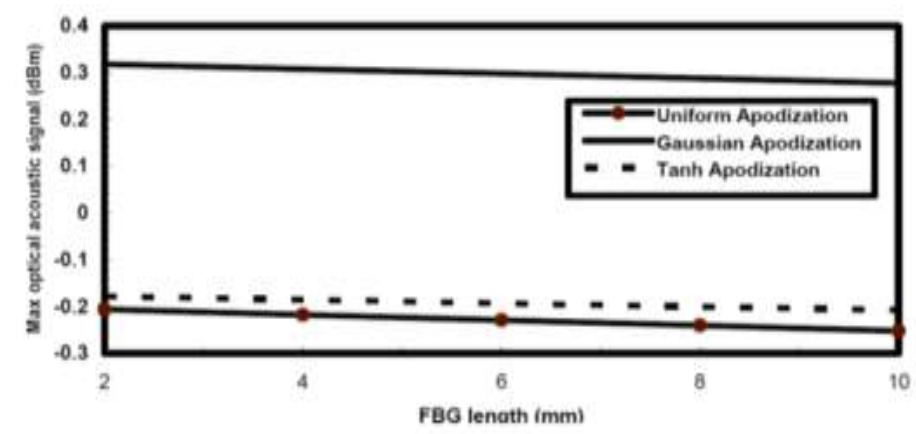

Figure 13. Max optical AS after FBG with FBG length for various FBG apodization functions at the central wavelength of $1.55 \mu \mathrm{m}$

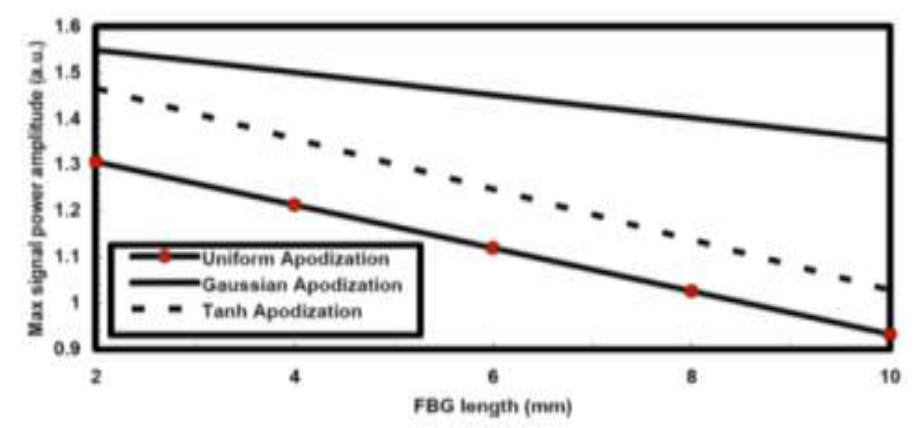

Figure 14. Max AS power amplitude after power combiner unit with FBG length for various FBG apodization functions at the central wavelength of $1.55 \mu \mathrm{m}$ 
Figure 15 clarifies the total AS power after power combiner unit with FBG length for various FBG $\mathrm{AF}$ at the central wavelength of $1.55 \mu \mathrm{m}$. The total AS power amplitude can be enhanced with FBG length of $2 \mathrm{~mm}$ for various FBG AF. Gaussian FBG AF has outlined better performance than other FBG AF. Figure 16 clarifies the max electrical power after power combiner unit with FBG length for various FBG AF at the central frequency of $193.1 \mathrm{THz}$. The max electrical power can be enhanced with FBG length of $2 \mathrm{~mm}$ for various FBG AF. Gaussian FBG AF has outlined better performance than other FBG AF.

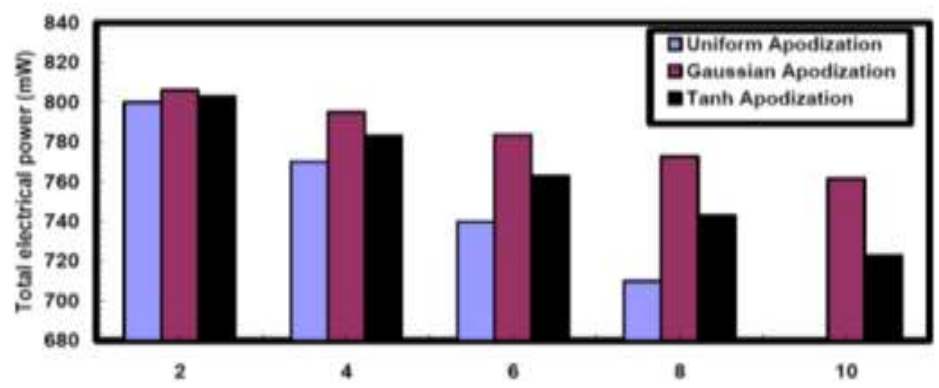

Figure 15. Total electrical power after power combiner unit with FBG length for various FBG apodization functions

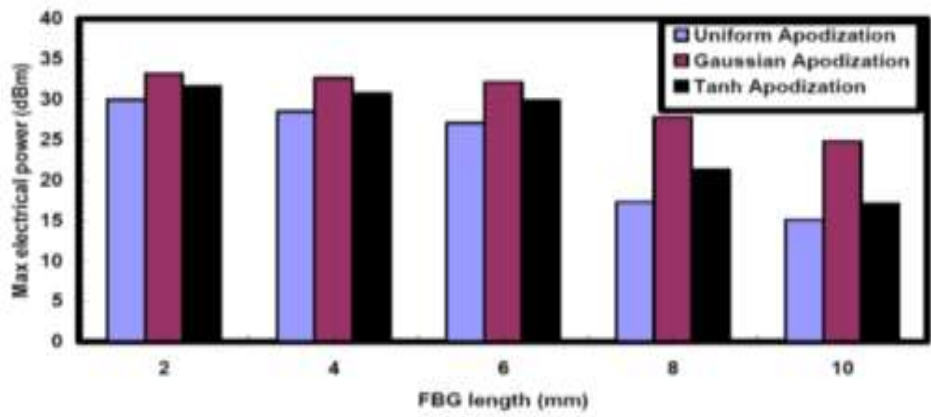

Figure 16. Max electrical power after power combiner unit with FBG length for various FBG apodization functions at the central frequency of $193.1 \mathrm{THz}$

\section{CONCLUSION}

We have been simulated the basic FBG apodization functions effects on the filtered optical AS. Max AS power amplitude after power combiner unit with FBG length for various FBG AF at the central wavelength of $1.55 \mu \mathrm{m}$. The max AS power amplitude can be enhanced with FBG length of $2 \mathrm{~mm}$ for various FBG AF. Gaussian FBG apodization function has outlined better performance than other FBG apodization functions. The total AS power amplitude can be enhanced with FBG length of $2 \mathrm{~mm}$ for various FBG AF. Gaussian FBG AF has outlined better performance than other FBG apodization functions THz. The max electrical power can be enhanced with FBG length of $2 \mathrm{~mm}$ for various FBG AF. Gaussian FBG AF has outlined better performance than other FBG AF. The AS power amplitude and optimized power at FBG length of $2 \mathrm{~mm}$ and at Gaussian FBG apodization function.

\section{REFERENCES}

[1] J. Witzens, et al., "Design of Transmission Line Driven Slot Waveguide Mach-Zehnder Interferometers and Application to Analog Optical Links," Optics Express., vol. 18, no. 16, pp. 1603-1628, 2010, doi: 10.1364/OE.18.016902.

[2] IS Amiri, et al., "Pump Laser Automatic Signal Control for Erbium-Doped Fiber Amplifier Gain, Noise Figure, and Output Spectral Power," Journal of Optical Communications, Published Online: 17 Dec. 2019, doi: 10.1515/joc-2019-0203.

[3] Ahmed Nabih Zaki Rashed, et al., "Transmission Performance Simulation Study Evaluation for High-Speed Radio Over Fiber Communication Systems," Wireless Personal Communications Journal, vol. 103, no. 2, pp. 1765-1779, Nov. 2018, doi: 10.1007/s11277-018-5879-y. 
[4] Ahmed Nabih Zaki Rashed, Mohammed Salah F. Tabbour, "Best candidate integrated technology for low noise, high speed, and wide bandwidth based transimpedance amplifiers in optical computing systems and optical fiber applications" International Journal of Communication Systems, vol. 31, no. 17, paper id: e3801, Nov. 2018, doi: $10.1002 /$ dac.3801.

[5] Mohamed Salah F. Tabbour, et al., "20 Gb/s Hybrid CWDM/DWDM for Extended Reach Fiber to the Home Network Applications," Proc. Natl. Acad. Sci., India, Sect. A Phys. Sci., vol. 89, no. 4, pp. 653-662, Oct.-Dec. 2019, doi: 10.1007/s40010-018-0526-2.

[6] C.S. Boopathi, et al., "Design of human blood sensor using symmetric dual core photonic crystal fiber," Results in Physics, vol. 11, pp. 964-965, Dec. 2018, doi: 10.1016/j.rinp.2018.10.065.

[7] S. Praveen Chakkravarthy, et al., "Ultra high transmission capacity based on optical first order soliton propagation systems,” Results in Physics, vol. 12, pp. 512-513, Mar. 2019, doi: 10.1016/j.rinp.2018.12.002.

[8] Ahmed Nabih Zaki Rashed, et al., "Transmittivity/Reflectivity, Bandwidth, and Ripple Factor Level Measurement for Different Refractive Index Fiber Grating Shape Profiles," Journal of Optical Communications, Published Online: 6 Feb. 2019, doi: 10.1515/joc-2018-0233.

[9] Ahmed Nabih Zaki Rashed, et al., "DWDM Channel Spacing Effects on the Signal Quality for DWDM/CWDM FTTx Network," Journal of Optical Communications, Published Online: 7 Feb. 2019, doi: 10.1515/joc-2019-0012.

[10] Ahmed Nabih Zaki Rashed, et al., "Numerical Analysis of Optical Properties Using Octagonal Shaped Photonic Crystal Fiber," Journal of Optical Communications, Published Online: 8 Feb. 2019, doi: 10.1515/joc-2019-0013.

[11] Ahmed Nabih Zaki Rashed, et al., "Different Graded Refractive Index Fiber Profiles Design for the Control of Losses and Dispersion Effects," Journal of Optical Communications, Published Online: 19 Feb. 2019, doi: 10.1515/joc-2019-0036.

[12] Ahmed Nabih Zaki Rashed, "Comparison between NRZ/RZ Modulation Techniques for Upgrading Long Haul Optical Wireless Communication Systems," Journal of Optical Communications, Published Online: 19 Feb. 2019, doi: 10.1515/joc-2019-0038.

[13] Ahmed Nabih Zaki Rashed, et al., "Nonlinear Characteristics of Semiconductor Optical Amplifiers for Optical Switching Control Realization of Logic Gates," Journal of Optical Communications, Published Online: 19 Feb. 2019, doi: 10.1515/joc-2019-0027.

[14] Kawsar Ahmed, et al., "Design of D-shaped elliptical core photonic crystal fiber for blood plasma cell sensing application," Results in Physics, vol. 12, pp. 2021-2025, Mar. 2019.

[15] T.V. Ramana, et al., "Numerical analysis of circularly polarized modes in coreless photonic crystal fiber," Results in Physics, vol. 13, Article 102140, June 2019, doi: 10.1016/j.rinp.2019.02.076.

[16] Ahmed Nabih Zaki Rashed, et al., "The switching of optoelectronics to full optical computing operations based on nonlinear metamaterials," Results in Physics, vol. 13, Article 102152, June 2019, doi: 10.1016/j.rinp.2019.02.088.

[17] S. Ranathive, et al., "Performance Signature of Optical Fiber Communications Dispersion Compensation Techniques for the Control of Dispersion Management," Journal of Optical Communications, Published Online: 5 March 2019, doi: 10.1515/joc-2019-0021.

[18] IS Amiri, et al., "Nonlinear Effects with Semiconductor Optical Amplifiers," Journal of Optical Communications, Published Online: 12 April 2019, doi: 10.1515/joc-2019-0053.

[19] IS Amiri, et al., "High-Speed Light Sources in High-Speed Optical Passive Local Area Communication Networks," Journal of Optical Communications, Published Online: 20 April 2019, doi: 10.1515/joc-2019-0070.

[20] Ahmed Nabih Zaki Rashed, et al., "Performance enhancement of overall LEO/MEO intersatellite optical wireless communication systems," International Journal of Satellite Communications and Networking, vol. 38, no. 1, pp. 31-40, Jan./Feb. 2020, doi: 10.1002/sat.1306.

[21] IS Amiri, et al., "Spatial Continuous Wave Laser and Spatiotemporal VCSEL for High-Speed Long Haul Optical Wireless Communication Channels," Journal of Optical Communications, Published Online: 24 April 2019, doi: 10.1515/joc-2019-0061.

[22] IS Amiri, et al., "Average Power Model of Optical Raman Amplifiers Based on Frequency Spacing and Amplifier Section Stage Optimization," Journal of Optical Communications, Published Online: 4 May 2019, doi: 10.1515/joc-2019-0081.

[23] IS Amiri, et al., "Temperature effects on characteristics and performance of near-infrared wide bandwidth for different avalanche photodiodes structures," Results in Physics, vol. 14, Article 102399, September 2019, doi: 10.1016/j.rinp.2019.102399.

[24] IS Amiri, Ahmed Nabih Zaki Rashed, "Simulative study of simple ring resonator-based brewster plate for power system operation stability," Indonesian Journal of Electrical Engineering and Computer Science (IJEECS), vol. 16, no. 2, pp. 1070-1076, November 2019, doi: 10.11591/ijeecs.v16.i2.pp1070-1076.

[25] S. Malathy, et al., "Upgrading Superior Operation Performance Efficiency of Submarine Transceiver Optical Communication Systems Toward Multi Tera Bit per Second," Computer Communications Journal, vol. 146, pp. 192-200, October 2019, doi: 10.1016/j.comcom.2019.08.009.

[26] IS Amiri, Ahmed Nabih Zaki Rashed, "Numerical investigation of V shaped three elements resonator for optical closed loop system," Indonesian Journal of Electrical Engineering and Computer Science (IJEECS), vol. 16, no. 3, pp. 1392-1397, December 2019, doi: 10.11591/ijeecs.v16.i3.pp1392-1397. 
[27] Ahmed Nabih Zaki Rashed, et al., "The effect of using different materials on erbium-doped fiber amplifiers for indoor applications," Results in Physics, vol. 15, paper id: 102650, Dec. 2019, doi: 10.1016/j.rinp.2019.102650.

[28] IS Amiri and Ahmed Nabih Zaki Rashed, "Power Enhancement of the U-shape Cavity Microring Resonator Through Gap and Material Characterizations," Journal of Optical Communications, Published Online: 19 Oct. 2019, doi: 10.1515/joc-2019-0108.

[29] IS Amiri, P et al., "The Engagement of Hybrid Ultra High Space Division Multiplexing with Maximum Time Division Multiplexing Techniques for High-Speed Single-Mode Fiber Cable Systems," Journal of Optical Communications, Published Online: 19 Oct. 2019, doi: 10.1515/joc-2019-0205.

[30] IS Amiri, Ahmed Nabih Zaki Rashed, "Signal Processing Criteria Based on Electro-Optic Filters for Fiber Optic Access Transceiver Systems," Journal of Optical Communications, Published Online: 12 Nov. 2019, doi: 10.1515/joc-2019-0116.

[31] IS Amiri, et al., "Pump Laser Automatic Signal Control for Erbium-Doped Fiber Amplifier Gain, Noise Figure, and Output Spectral Power," Journal of Optical Communications, Published Online: 17 Dec. 2019, doi: 10.1515/joc-2019-0203.

[32] IS Amiri, Ahmed et al., "Performance Enhancement of Fiber Optic and Optical Wireless Communication Channels by Using Forward Error Correction Codes," Journal of Optical Communications, Published Online: 24 Dec. 2019, doi: 10.1515/joc-2019-0191.

[33] Mahmoud M. A. Eid, et al., "Mono Rectangular Core Photonic Crystal Fiber (MRC-PCF) for Skin and Blood Cancer Detection," Plasmonics Journal, Published Online: 20 November 2020, doi: 10.1007/s11468-020-01334-0.

[34] Mahmoud M. A. Eid, et al., "Best selected optical fibers with wavelength multiplexing techniques for minimum bit error rates," Journal of Optical Communications, Dec. 2020, doi: 10.1515/joc-2020-0239.

[35] Mahmoud M. A. Eid, et al., "The influence of electrical filters with sequence generators on optical ISL performance evolution with suitable data rates," Journal of Optical Communications, Published Online: 17 December 2020, doi: 10.1515/joc-2020-0257.

[36] Aadel M. Alatwi, et al., "Wavelength division multiplexing techniques based on multi transceiver in low earth orbit intersatellite systems," Journal of Optical Communications, Published Online: 29 June 2020, doi: 10.1515/joc-2019-0171.

[37] Hazem M. El-Hageen, et al., "Different modulation schemes for direct and external modulators based on various laser sources," Journal of Optical Communications, Published Online: 25 July 2020, doi: 10.1515/joc-2020-0029.

[38] Hazem M. El-Hageen, et al., "High-speed signal processing and wide band optical semiconductor amplifier in the optical communication systems," Journal of Optical Communications, Published Online: 3 August 2020, doi.org/10.1515/joc-2020-0070.

[39] Aadel M. Alatwi, et al., "Beam divergence and operating wavelength bands effects on free space optics communication channels in local access networks," Journal of Optical Communications, Published Online: 8 August 2020, doi: doi.org/10.1515/joc-2019-0276.

[40] Hazem M. El-Hageen, et al., "Laser measured rate equations with various transmission coders for optimum of data transmission error rates," Indonesian Journal of Electrical Engineering and Computer Science (IJEECS), vol. 20, no. 3, pp. 1406-1412, Dec. 2020, doi: 10.11591/ijeecs.v20.i3.pp1406-1412.

[41] Mahmoud M. A. Eid, et al., "Highly sensitive nonlinear photonic crystal fiber based sensor for chemical sensing applications," Microsystem Technologies Journal, doi.org/10.1007/s00542-020-05019-w, Published Online: 5 September 2020.

[42] Mahmoud M. A. Eid, et al., "Fabry Perot laser properties with high pump lasers for upgrading fiber optic transceiver systems," Journal of Optical Communications, Published Online: 21 September 2020, doi: 10.1515/joc2020-0146.

[43] Mahmoud M. A. Eid, et al., "Spatial optical transceiver system-based key solution for high data rates in measured index multimode optical fibers for indoor applications," Journal of Optical Communications, Published Online: 21 September 2020, doi: 10.1515/joc-2020-0117.

[44] Mahmoud M. A. Eid, et al., "Simulation study of signal gain optimization based on hybrid composition techniques for high speed optically dense multiplexed systems," Journal of Optical Communications, Published Online: 22 September 2020, doi: 10.1515/joc-2020-0150.

[45] Mahmoud M. A. Eid, et al., "High speed optical switching gain based EDFA model with $30 \mathrm{~Gb} / \mathrm{s}$ NRZ modulation code in optical systems," Journal of Optical Communications, Published Online: 21 October 2020, doi: 10.1515/joc-2020-0223.

[46] Mahmoud M. A. Eid, et al., "Fast speed switching response and high modulation signal processing bandwidth through LiNbO3 electro-optic modulators," Journal of Optical Communications, Published Online: 27 October 2020, doi: 10.1515/joc-2020-0012.

[47] Mahmoud M. A. Eid, et al., "Performance enhancement of transceiver system based inter satellite optical wireless channel (IS-OWC) for ultra long distances," Journal of Optical Communications, Published Online: 27 October 2020, doi: 10.1515/joc-2020-0216.

[48] Mahmoud M. A. Eid, et al., "Simulation performance signature evolution of optical inter satellite links-based booster EDFA and receiver preamplifiers," Journal of Optical Communications, Published Online: 27 October 2020, doi: 10.1515/joc-2020-0190. 
[49] Mahmoud M. A. Eid, et al., "Influence of dense wavelength division multiplexing (DWDM) technique on the low earth orbit intersatellite systems performance," Journal of Optical Communications, Published Online: 6 November 2020, doi: 10.1515/joc-2020-0188.

[50] Mehtab Singh, Jyoteesh Malhotra, "Performance investigation of high-speed FSO transmission system under the influence of different atmospheric conditions incorporating 3-D orthogonal modulation scheme," Optical and Quantum Electronics, vol. 51, Article no. 285, 2019, doi: 10.1007/s11082-019-1998-2.

[51] Mehtab Singh, Jyoteesh Malhotra, " $4 \times 20$ Gbit/s-40 GHz OFDM based Radio over FSO transmission link incorporating hybrid wavelength division multiplexing-mode division multiplexing of LG and HG modes with enhanced detection," Optoelectronics and Advanced Materials-Rapid Communications, vol. 14, no. 5-6, pp. 233-243, 2020.

[52] Mehtab Singh, Jyoteesh Malhotra, " 40 Gbit/s- 80 GHz hybrid MDM-OFDM-Multibeam based RoFSO transmission link under the effect of adverse weather conditions with enhanced detection," Optoelectronics and Advanced Materials-Rapid Communications, vol. 14, no. 3-4, pp. 146-153, 2019.

[53] Mehtab Singh, Jyoteesh Malhotra, " $2 \times 10 \mathrm{Gbit} / \mathrm{s}-10 \mathrm{GHz}$ Radio over Free Space Optics Transmission System Incorporating Mode Division Multiplexing of Hermite Gaussian Modes," Journal of Optical Communications, 2019, doi: 10.1515/joc-2019-0047.

[54] D. A. J. Al-Khaffaf and H. S. R. Hujijo, "High data rate optical wireless communication system using millimeter wave and optical phase modulation," ARPN Journal of Engineering and Applied Sciences, vol. 13, no. 23, pp. 9086-9092, Dec. 2018.

[55] Mehtab Singh, et al., "Performance evaluation of 6.4 Tbps dual polarization quadrature phase shift keying Nyquist-WDM superchannel FSO transmission link: Impact of weather conditions," Alexandria Engineering Journal, vol. 59, no. 2, pp. 977-986, 2020, doi: 10.1016/j.aej.2020.03.031.

[56] Abd El-Naser A. Mohamed, et al., "Different Pumping Categories of Erbium Doped Fiber Amplifiers Performance Signature With Both Wide Multiplexing and Modulation Techniques," International Journal of Science, Engineering and Technology Research (IJSETR), vol. 5, no. 3, pp. 622-642, March 2016.

[57] Ibrahim M. El-Dokany, et al., "Optical Preamplifier and Inline Amplifiers Comparison Based Raman-EDFA Hybrid Amplifiers in 40×10 Gb/s DWDM System," Minufiya J. of Electronic Engineering Research (MJEER), vol. 25, no. 2, pp. 147-160, July 2016, doi: 10.21608/mjeer.2016.64093.

[58] Ahmed M. El-Eraki, et al., "Fast Routing Algorithm in Optical Multistage Interconnection Networks using Fast Window Method," International Journal of Advanced Research in Electronics and Communication Engineering (IJARECE), vol. 6, no. 1, pp. 37-43, Jan. 2017. 